\title{
Análisis de la sentencia Cs Rol 12169-2017 a la luz de la pérdida de la chance $^{*}$
}

\section{Sonia Inostroza Adasme**}

Resumen. El maremoto del año 2010 en Chile registró el mayor número de víctimas fatales asociado a este tipo de desastres en los últimos treinta años en Suramérica ${ }^{1}$. Por ello, los familiares de un número considerable de personas demandaron al Fisco de Chile. Este trabajo analiza la decisión del tribunal $a q u o^{2}$, de la cual no se pronuncia el tribunal ad quem ${ }^{3}$, pero sí lo hace el tribunal de casación, que hacen alusión a la llamada "pérdida de la chance".

PALABRAS ClAVE: maremoto, víctimas fatales, indemnizar perjuicios, pérdida de la chance.

* Fecha de recepción: 15 de octubre de 2019. Fecha de aceptación: 27 de abril de 2020 .

Para citar el artículo: Inostroza AdAsme, S., "Análisis de la sentencia cs Rol 12169-2017 a la luz de la pérdida de la chance", Revista de Derecho Privado, n. ${ }^{\circ}$ 39, julio-diciembre 2020, 409-423, DoI: https://doi.org/10.18601/01234366.n39.17.

** Universidad de los Andes, Santiago de Chile, Chile; doctoranda en derecho. Magíster en derecho y Abogada, Pontificia Universidad Católica de Valparaíso, Valparaíso, Chile. Contacto: sainostroza@ miuandes.cl. Orcid: 0000-0002-2437-4782.

1 Contreras, M. y Winckler, P., "Pérdidas de vidas, viviendas, infraestructura y embarcaciones por el tsunami del 27 de febrero de 2010 en la costa central de Chile". Obras y proyectos, 14, 2013, 6, doi: [https://dx.doi.org/10.4067/S0718-28132013000200001].

222 Juzgado Civil de Santiago, Rol: 21111-2013.

3 Corte de Apelaciones de Santiago, Rol: 8013-2016. 


\title{
The Court Judgment Analysis (Rol 12169-2017) According to the Loss of Chance
}

\begin{abstract}
The 2010 tsunami has recorded the highest number of fatalities associated with these natural disasters in South America ${ }^{4}$ in the last thirty years. Faced with such events and the subsequent death of a considerable number of people, their families sued the Chilean Treasury. I will comment the decision of the tribunal $a q u o^{5}$, of which the tribunal ad quem ${ }^{6}$ does not pronounce, but the tribunal of cassation does. These iudicata allude to loss of chance.
\end{abstract}

KEYwords: tsunami, fatalities, compensating damages, loss of chance.

Sumario: i. El caso Escalona y otros con fisco de Chile cs Rol 12169-2010. II. La demanda. III. La contestación. Iv. El fallo. v. La pérdida de la chance como partida indemnizatoria en el caso sub lite. Consideraciones finales. Referencias.

\section{El caso Escalona y otros con fisco de Chile cs Rol 12169-2010}

Una serie de personas presenta demanda con la pretensión de que se declare la responsabilidad del Estado por falta de servicio, que hacen consistir en la circunstancia de que los organismos pertinentes - la Oficina Nacional de Emergencia del Ministerio del Interior (ONEMI) y el Servicio Hidrográfico y Oceanográfico de la Armada de Chile (SHOA) - no comunicaran de manera oportuna la alerta de tsunami el 27.02.2010 ${ }^{[7]}$.

El tribunal a quo acogió la demanda y tanto el tribunal ad quem como el de casación la confirman.

Lo interesante del fallo para nuestros fines es analizar la figura indemnizatoria a la que recurren el tribunal de primera instancia y la Corte Suprema para resarcir el daño a las víctimas.

\section{La demanda}

Los hechos que dan lugar a la acción se configuran en el terremoto y posterior maremoto del año 2010.

4 Contreras, M., y WincKler, P., "Pérdidas de vidas, viviendas, infraestructura y embarcaciones por el tsunami del 27 de febrero de 2010 en la costa central de Chile", Obras y proyectos, 14, 2013, 6-19, doi: [https://dx.doi.org/10.4067/S0718-28132013000200001].

522 Juzgado Civil de Santiago, Rol: 21111-2013.

6 Corte de Apelaciones de Santiago, Rol: 8013-2016.

7 Farfarello Galletti, A. F., Responsabilidad del Estado: terremoto del 27 de febrero de 2010, Santiago de Chile, Rubicón Editores, 2019, 273 ss. 
La acción impetrada la realizaron 74 personas debidamente representadas en contra del Fisco de Chile representado por el presidente del Consejo de Defensa del Estado. En el libelo de la demanda se pretende una condena al pago total de diez mil seiscientos millones de pesos, más reajustes e intereses, por concepto de daño moral.

Los actores fundan su petición en que sufrieron graves perjuicios ocasionados por la falta de servicio de servidores públicos, durante el terremoto ocurrido el día 27 de febrero de 2010, para lo cual distinguen de acuerdo con la zona geográfica en que se encontraban al momento de los acontecimientos.

Cabe destacar que los demandantes tienen la condición de ser víctimas por rebote o repercusión. Y esta calidad la obtienen en el contexto del hecho que originó el desastre, toda vez que las víctimas iniciales han muerto. Así las cosas, "el daño sufrido por persona diversa del sujeto inmediatamente perjudicado siendo generalmente familiares que dependen económicamente de él, o que se han vinculado comercial o profesionalmente con ella dan lugar al denominado daño por rebote o repercusión"».

\section{La contestación}

La demandada contestó la acción dirigida en su contra, solicitando su íntegro rechazo, y señala que los demandantes han descontextualizado los hechos, pues ellos se encuentran dentro del ámbito de la conmoción, caos y destrucción masiva.

Realizan un detallado examen de los acontecimientos de aquella madrugada para demostrar que no incurrieron en falta de servicio.

A continuación, oponen la excepción de caso fortuito o fuerza mayor aludiendo a estas como causa de eximente de responsabilidad. Hacen hincapié en que tanto el terremoto como el maremoto son hechos clásicos de casos fortuitos según lo establece el artículo 45 del Código de Bello 9 .

Agrega que las circunstancias de imprevisibilidad e irresistibilidad son reconocidas por la doctrina como determinantes para la configuración de la fuerza mayor, y que en el caso de autos concurren los requisitos para la configuración de esta excepción.

\section{EI fallo}

\section{A. Primera instancia}

El tribunal de primera instancia trata de forma detallada cada uno de los temas que han sido objeto del caso de marras.

8 Elorriaga de Bonis, F., "Del daño por repercusión o rebote”. Revista Chilena de Derecho, vol. 26, n. ${ }^{\circ} 2,1999,369$.

9 Artículo 45, Código Civil: "Se llama fuerza mayor o caso fortuito el imprevisto a que no es posible resistir, como un naufragio, un terremoto, el apresamiento de enemigos, los actos de autoridad ejercidos por un funcionario público, etc.”. 
No obstante, respecto de nuestra materia en estudio realiza las siguientes observaciones en el considerando cuadragésimo séptimo: “[...] que respecto de la imprevisión del hecho ésta solo puede considerarse acerca del terremoto, pero no así del maremoto. Ello debido a que se asentó en el caso sub lite, la omisión de una alerta roja que correspondía haber emitido la ONEMI, a la luz de los acontecimientos, ya que, si bien la información difundida fue errónea, no se puede discutir que no haya sido adecuada a base de los datos que se contaban en el momento. Es por este motivo que pierde vigor la alegación de imprevisibilidad. Para comprender mejor este punto se construye la siguiente situación: el SHOA decretó alerta de tsunami a las 03:51 am y la remitió por fax al CAT de la ONEMI a las 04:07 am, apoyado en el boletín del Centro de Alerta de Tsunamis del Pacífico recibido a las 03:48 am, que indicaba probabilidad de tsunami. Ante esta situación ¿dónde se encuentra la imprevisibilidad del hecho?"10.

Con todo, la alerta emitida por el sHOA solo fue cancelada a la 05:10 am, por lo que en el tiempo que media entre recibir la alerta y erróneamente cancelarla varias olas habían azotado las costas chilenas, y en ello se aprecia una conducta culposa. Por consiguiente, era absolutamente previsible la ocurrencia de tsunami. Lo anterior hace concluir al sentenciador que el actuar constituye una omisión culposa y no un caso fortuito o fuerza mayor.

Sin embargo, más relevancia tiene aún para nuestro trabajo lo plasmado en el considerando quincuagésimo segundo "de los perjuicios indemnizables".

Parte el relato señalando que la acción indemnizatoria impetrada corresponde a la derivada por responsabilidad extracontractual. Agrega que en este caso el Fisco será objeto de la aplicación de las disposiciones contenidas en los artículos 2314 y siguientes del Código de Bello, puesto que las personas jurídicas tienen capacidad suficiente para ser sujetos pasivos de este tipo de responsabilidad. Continúa:

Corresponde analizar la existencia de perjuicios indemnizables y, de ser procedente, fijar su cuantía.

La falta de servicio ya comentada generó a las víctimas la pérdida de una oportunidad, la que en el caso de autos corresponde a la pérdida de la posibilidad de sobrevida, no solo de haber contado con un sistema de alerta eficiente, que sobrellevara el colapso que importa un sismo no determinable, pero sí previsible (por la condición sísmica del suelo chileno), de haber mediado el aviso y alerta de tsunami por las autoridades correspondientes, en forma oportuna.

Lo anterior se enmarca en la doctrina que sitúa este tipo de daño, entre el daño eventual y el lucro cesante, así lo ha entendido además la Iltma. Corte de Apelaciones de Concepción, según fallo de fecha 12 de enero del año 2000 en Rol 167-1999: “entre el daño cierto y el daño eventual, existe una zona intermedia, gris, que es la 
probabilidad suficiente, la cual es más que la posibilidad pero menos que la certeza, situación que se conoce en el derecho como chance".

Para el autor nacional, abogado Mauricio Tapia Rodríguez, ante la presencia de una pérdida de oportunidad, corresponde al juez la determinación del monto de la indemnización, ya que de esta manera se respetaría el principio de igualdad ante la ley.

Debiéndose reparar dicha pérdida, según este autor, siempre que se den los siguientes requisitos: (1) el interés lesionado debe ser legítimo y relevante; (2) el perjuicio provocado a dicho interés debe ser directo; (3) el perjuicio provocado debe afectar personalmente a la víctima; y (4) el perjuicio reparable debe ser cierto. De este modo, la pérdida de una chance implica necesariamente la comprobación de un acto ilícito previo, que en el caso de marras se configura por la infracción a los protocolos de emergencia ya señalados, y un vínculo causal.

Existen sentencias que recogen lo precedente, así en fallo de la Excma. Corte Suprema en Rol 29365-14, en su considerando $14^{\circ}$ indica: "estos sentenciadores han llegado al convencimiento de que, como consecuencia de la falta de servicio atribuida al demandado, al señor Vásquez Velásquez, no se le privó de la vida sino que de la oportunidad de luchar dignamente por ella"11.

Finalmente, en el considerando quincuagésimo tercero, número II, señala que acoge la demanda de indemnización de perjuicios y que condena a la demandada a pagar unas sumas que allí mismo especifica.

\section{B. Segunda instancia}

El tribunal de apelación establece claramente en sus consideraciones que la responsabilidad en el caso de marras "radica en la falta de servicio oportuno de una parte de las autoridades respectivas, en orden a [sic] prevenir, a quienes podían verse afectados por los efectos del tsunami que se anunciaba y que posteriormente azotó las costas de Chile $[\ldots] " 12$. Cabe destacar que el tribunal no se pronuncia de forma directa respecto de la "pérdida de la chance" y deja confirmada la sentencia del tribunal a quo.

\section{Casación}

La Corte Suprema aborda el tema de la chance a propósito del requisito de la causalidad, toda vez que para que exista falta de servicio por parte de los entes del Estado debe existir una relación de causalidad entre el hecho generador y el daño producido.

12 Corte de Apelaciones de Santiago, Rol 8013-2016, considerando número 1. 
Así las cosas, distingue dos elementos que son integrantes de la relación de causalidad: (1) el elemento natural: en éste se puede establecer que un hecho es condición necesaria de un cierto resultado cuando de no haber existido la condición, el resultado tampoco se habría producido; (2) el elemento objetivo: en éste es indispensable que el daño producido pueda imputarse normativamente al hecho ilícito.

Por consiguiente, una vez determinada la causalidad natural habrá que determinar si el daño puede ser atribuible a la conducta determinada. El problema que subyace en el caso sub lite es que la certidumbre sobre la relación causal es difícil de establecer, por lo que en estos regímenes de responsabilidad en la mayoría de los casos solo será posible efectuar una estimación de la probabilidad de que el daño se deba a un hecho u omisión por el cual el demandado deba responder. Es así como en el caso en concreto existen dificultades para establecer el vínculo causal. Sin embargo, los sentenciadores acuden acertadamente a la teoría de la pérdida de la chance como mecanismo de solución de problemas de incerteza causal ${ }^{13}$. Concretamente se refieren a esta moderna forma de indemnización en los considerandos $12 .^{\circ}$ y $13 .{ }^{\circ}$.

El razonamiento duodécimo reproduce el problema acerca de la causalidad que acabamos de exponer.

Con todo, será el considerando decimotercero el determinante para nuestro estudio. Los sentenciadores señalan nuevamente la incertidumbre que presenta el vínculo causal en los hechos que dan pie a la discusión. Sin embargo, establecen que habrá que atender al hecho generador de la responsabilidad y éste no es el terremoto, sino la omisión de comunicar la alerta de tsunami, por lo cual concluyen que habrá que acudir a la teoría de la pérdida de chance.

De esta manera, hacen hincapié en lo plasmado por el autor nacional Mauricio Tapia: "La pérdida de la chance se encuentra entre estas últimas hipótesis (cuando no se sabe lo que habría ocurrido en el futuro de no haberse cometido el hecho ilícito), esto es, incide en la frustración de una expectativa de obtener una ganancia o de evitar una pérdida. Pero, a diferencia del daño eventual, en los casos de daño eventual puede concluirse que efectivamente la víctima tenía oportunidades serias de obtener el beneficio esperado o de evitar el perjuicio, tal como ya se ha mencionado"; destaca enseguida que se trata del caso de "una víctima que tenía oportunidades de obtener un bien aleatorio que estaba en juego (ganar un proceso, recobrar la salud, cerrar un negocio, acceder a una profesión, etc.) y el agente, al cometer el hecho ilícito, destruyó ese potencial de oportunidades (olvidó apelar, no efectuó un examen, omitió certificar un documento, lesionó al postulante, etc...). La víctima en todos estos casos se encontraba inmersa en un proceso que podía arrojarle un beneficio o evitarle una pérdida (tratamiento médico, apelación de una sentencia, preparación de un examen, etc.) y el agente destruyó por completo con su negligencia las chances que la víctima tenía para lograr tal ventaja"14. Agregan los sentenciadores: 
El vínculo de causalidad, se relaciona estrechamente con la teoría en análisis, pues aplicando las ideas expuestas en los considerandos anteriores se concluye que la relación causal se vincula con la circunstancia de privar a las víctimas de una oportunidad de poner a resguardo su vida, cuestión que efectivamente podrían haber realizado si hubiesen recibido la información de riesgo de tsunami oportunamente.

La omisión de la entrega oportuna de la información solo puede relacionarse causalmente con la pérdida de la oportunidad de alejarse de las zonas de riesgos antes de la llegada de las olas que determinan la muerte o afectación de las personas, cuestión que a su turno se relaciona con la opción que tienen aquellos que demandan en su calidad de víctimas por repercusión o rebote de haber contado por más tiempo con su ser querido.

En virtud de lo razonado, no es posible asumir la falta de relación de causalidad esgrimida por el recurrente, toda vez que en caso alguno los sentenciadores han señalado que el vínculo de la omisión es en relación a la muerte, pues es una obviedad que la muerte se relaciona directamente con un hecho de la naturaleza ${ }^{15}$.

Finalmente, por estas consideraciones el tribunal rechaza el recurso de casación en el fondo deducido por la parte demandada.

\section{La pérdida de la chance como partida indemnizatoria en el caso sub lite}

El caso que suscita la presente sentencia guarda estrecha relación con aquella categoría jurídica denominada ordinariamente a través de un galicismo, como "chance": nosotros, animados por un propósito de corrección filológica, la designaremos sin más con el vocablo "oportunidad". La diferencia etimológica no incide tanto, por lo demás, en su significado: chance, o choir, en francés, se deriva del verbo latino cado, caer, del cual proviene también cadentia, que se puede traducir no solo como "caído", sino también como "posibilidad real de éxito", tal como sucede en la música cuando un acorde disonante se resuelve sobre otro consonante. "Oportunidad" procede del latín "oportunitas -atis", que es la sazón, coyuntura o conveniencia de tiempo y de lugar. La palabra está compuesta por el prefijo op, por "ob", delante, y el nombre portunus, forma de portus, puerto: lo que está a la entrada (delante) del puerto, en situación cómoda y ventajosa ${ }^{16}$. Así, tener una oportunidad no consiste en

14 TAPIA Rodríguez, M., "Pérdida de la chance: ¿un perjuicio indemnizable en Chile?”, en ElorRiaga DE Bonis, F. (coord.), Estudios de Derecho Civil VII, Jornadas Nacionales de Derecho Civil, Viña del Mar, 2011, Legal Publishing Chile, 650 .

15 Considerando décimo tercero, párrafos 4,5 y 6

16 BÁrcia, R., Diccionario General Etimológico de la lengua española, voz “oportuno", "oportunidad”, 1881 . 
la vaga y genérica posibilidad de obtener un bien o evitar un mal, sino en una opción específica, seria y real respecto de aquello. Desarrollaremos más adelante este concepto. El problema de la pérdida de oportunidad, aparte algún antecedente romano que podemos encontrar, por ejemplo, en un texto de $\mathrm{Neracio}^{17}$, tiene data reciente en nuestro derecho y se puede remontar su origen, en la tradición continental, a un fallo de la Corte de Casación francesa del año $1889^{[18]}$, donde se condenó a un funcionario ministerial por cuanto su conducta negligente había impedido la prosecución de un juicio, por lo cual el actor perdió la oportunidad de ganar el pleito. Para la familia jurídica del common law, más que a través del Reino Unido, acudimos a la jurisprudencia estadounidense, donde, en 1917, la Corte de Apelaciones de Texas condenó a indemnizar la oportunidad perdida de ganar un premio en un concurso de animales a un transportista que retrasó la llegada de uno de los caballos concursantes ${ }^{19-20}$.

Tal como lo hemos advertido, la oportunidad, como bien jurídico, no describe "una posibilidad genérica y vaga, que no implica sino un daño puramente hipotético o eventual" 21 ; la noción que analizamos no significa la simple esperanza de un suceso favorable, como podría ser, por ejemplo, la obtención del premio mayor de la lotería, sino una probabilidad que la doctrina ha caracterizado con las expresiones "real" y "seria": es seria cuando la probabilidad del suceso favorable va más allá de la simple esperanza y aunque no sea seguro -pues la oportunidad supone siempre un resultado aleatorio- existen proporcionalmente buenas posibilidades de éxito; es real cuando el titular de la oportunidad se encuentra en una situación fáctica o jurídica idónea para aspirar a la obtención de las metas que perseguía ${ }^{22}$. Estos rasgos,

17 D.9.2.23. Se refiere al que pierde la oportunidad de aceptar una herencia, y como consecuencia, la ventaja que significa su adquisición.

18 Citado por Tapia Rodríguez, M., op . cit. nota n. ${ }^{\text {2: }}$ "Su reparación es admitida ampliamente por la doctrina y jurisprudencia francesa. Véase por ejemplo: AUBRY et RAU, Cours de droit civil français, Responsabilité délictuelle, t. vI-2, por N. Dejean de la Bâtie, 8. ${ }^{a}$ ed. sous la direction de André Ponsard et Ibrahim Fadlallah, Paris, Libraires Techniques, 1989, 36 y s.; Jean Carbonnier, Droit Civil, Les obligations, t. 4, París, PUF, Thémis Droit Privé, 2000, 22. a edición refundida, 378 y s.; JAQUES Flour, Jean-Luc Aubert et Eric Savaux, Droit civil, Les obligations, 2. Le fait juridique, París, Armand Colin, 2001, 9. ${ }^{\text {a }}$ ed., 126 y s.; PhilipPe Le TourneAu y LoḮ CADIET, Droit de la responsabilité et des contrats, París, Dalloz Action, 2002-2003, 371 y s.; PhilipPe Malaurie y Laurent Aynès, Obligations. Responsabilité delictuelle, París, Cujas, 2001-2002, 11. a ed., 139 y s.; Henri, Léon y Jean Mazeaud, y François Chabas, Leçons de droit civil, Obligations, Théorie générale, t. in, 1 v., París, Montchrestien, 1998, 9. a edición por François Chabas, 416 y s.; Boris Starck, Henri Roland y Laurent Boyer, Obligations, 1. Responsabilité délictuelle, París, Litec, 1996, 5. ed., 61 y s.; François Terré, PhilipPe Simler y Yves Lequette, Droit civil, Les obligations, París, Précis Dalloz, 2002, 8. ${ }^{\text {a }}$ ed., 679 y s.; Geneviève Viney y Patrice Jourdain, Les conditions de la responsabilité, Traité de droit civil, sous la direction de Jacques Ghestin, París, LGDJ, 1998, 2. ed., 71 y s.”.

19 Trigo Represas, F. A., Pérdida de chance, Astrea, Argentina, 2008, 28-29.

20 Para las más antiguas sentencias, véase: TAPIA RodríGUEZ, M., "Pérdida de una chance. Su indemnización en la jurisprudencia chilena", Revista de Derecho, n. ${ }^{\circ} 2,2012,251-252$.

21 Trigo Represas, F. A., op. cit., 30.

22 Chaia, R. A. y Prévôt, J. M., Pérdida de chance de curación, Editorial Astrea, primera edición, Argentina, 2007, p. 45. 
de seriedad y realidad, pues, diferencian a la oportunidad de una simple spes o esperanza, y también de la pérdida de ella con el daño que conocemos bajo el nombre de "eventual". La profesora María del Carmen Crespo Mora califica la pérdida de oportunidad como una categoría general de daños en la que se incluyen "todos aquellos casos en los que el agente dañante, con su actuación u omisión ha interrumpido un proceso con el que la víctima tenía posibilidades de conseguir una ganancia o evitar una pérdida, por encontrarse en la situación idónea para ello": el concepto incluye el elemento de la seriedad en cuanto alude a la "posibilidad de conseguir la ganancia", la cual entendemos que ha de ser verosímil, y también incluye el elemento de la realidad, ya que la víctima ha de encontrarse "en una situación idónea para ello"23.

En el caso que analizamos, efectivamente, de haber operado convenientemente el servicio de la administración pública, a través del SHOA y la ONEMI, las víctimas del maremoto habrían tenido la real oportunidad de salvar sus vidas, una opción altísimamente probable, que supera con largueza el concepto de simple esperanza, y además, se encontraban en situación de hacerlo, tanto desde el punto de vista fáctico como desde el jurídico.

Es necesario saber distinguir la ventaja autónoma que significa la oportunidad, frente a aquel otro bien que se espera como consecuencia de haber tenido dicha oportunidad: la oportunidad misma constituye un bien cierto y actual; la ventaja que se espera como consecuencia de ella es, aunque probable, incierta y eventual: en el caso sub iudice los demandantes reclaman contra el Fisco por cuanto la falta de servicio de los entes estatales privó a las víctimas directas de la oportunidad que consistía en colocarse a salvo del maremoto, y tal era un bien cierto y actual en el momento de la omisión atribuida a el SHOA y la ONEMI. Como consecuencia de esa imposibilidad de aprovechar la oportunidad, las víctimas encontraron la muerte, pero ciertamente podemos decir que en aquel momento la posibilidad de sobrevivencia era un bien eventual y futuro. De igual manera en el ya mencionado caso resuelto por la Corte de Apelaciones de Texas, el bien que se reclama no es el premio que hubiera podido ganar el animal que concursaba, sino la posibilidad de participar en el concurso.

La oportunidad, pues, en concepto del profesor Tapia Rodríguez ${ }^{24}$, “es la posibilidad de acceder a la obtención de un bien que en sí es aleatorio, pero del que existen razonables expectativas de obtenerlo: ganar un proceso, cerrar un negocio, recobrar la salud, evitar la muerte, acceder a una profesión, etc.”, pero de manera tal que la privación de esa oportunidad significará a la víctima perder una ocasión no difusa, sino específica y probable, de quedar en mejores condiciones de las que detentaba en el momento de producirse esa pérdida.

Una clara distinción entre el bien actual que significa la oportunidad propiamente tal y el bien aleatorio esperado como consecuencia de haberla aprovechado nos 
llevará a un análisis más adecuado relativo a los muy pertinentes problemas de la causa y del daño.

Consideremos ante todo la cuestión del daño. Como ha señalado el profesor Barros Bourie ${ }^{25}$, la doctrina exige que el daño reparable sea cierto, y tal requisito resulta del todo evidente cuando lo que se reclama es un daño pasado, como sucede, por ejemplo, con el daño emergente. En el caso que estudiamos, claro está, aquel que se podría llamar el daño definitivo, el que es una consecuencia de la pérdida de oportunidad, no existe necesariamente en el momento de la sentencia, pero debemos tener siempre presente que la cuestión a que se refiere el caso no es el que hemos denominado daño definitivo sino el específico daño que consiste en perder una oportunidad: por eso se debe exigir certidumbre respecto de la conducta del agente que causa el daño productor de la pérdida de oportunidad, entendida ésta como un bien en sí mismo, diferente del que deriva de su aprovechamiento. La posibilidad que tenían las víctimas directas para salvarse del maremoto es un bien en sí, tiene una "entidad ontológica" como argumentan quienes estudian el presente problema desde la perspectiva del daño, aparte de la innegable ventaja de continuar con vida, a la que, desde el punto de vista de la finalidad, se orienta la oportunidad de tomar precauciones. Es esta una entidad patrimonial preexistente al hecho ilícito, cuya indeterminación puede incidir en la certeza respecto del resultado final, pero no respecto de su real existencia y de la conducta del agente causante.

Pese a sostener el ya referido tratamiento ontológico, y en la medida en que lo hagamos, la causalidad adecuada para el caso se ha de establecer entre la acción u omisión del agente que causó el daño -en nuestro ejemplo, la falta de información debida a negligencia o incompetencia de los organismos públicos- y el resultado de causar esta omisión la pérdida de oportunidad para las víctimas directas de ponerse a buen resguardo del maremoto y así salvar sus vidas ${ }^{26}$. Verdaderamente, y tal como lo sostienen Chabas y Trigo ${ }^{27}$, la relación de causalidad debe existir entre el hecho ilícito lesivo - la falta de aviso en nuestro caso- y la oportunidad que se frustra: es verdad que tal oportunidad lleva ínsita la incertidumbre del perjuicio definitivo, y por tal razón los tratadistas procuran establecer una relación valórica cuantitativa, entre dicha pérdida de oportunidad y el resultado adverso final, y para cubrir esa distancia y diferencia, por cuanto no hay certeza absoluta sobre que al faltar el acceso a la oportunidad, y precisamente por esta circunstancia, se materialice finalmente un resultado adverso, "han surgido en el derecho comparado los llamados umbrales o estándares de certidumbre, técnica que permite salvar estas situaciones especialmente complejas"28. En ausencia de un pronóstico certero sobre la ocurrencia del "daño 2007, 236-237. 
definitivo", se procura efectuar un juicio de probabilidad, y conforme a semejante técnica, daríase por establecida la relación causal entre el hecho ilícito y el daño: de todas maneras, ese nexo no puede sino ser más frágil respecto de la que podríamos denominar causalidad natural.

\section{A. Quantum indemnizatorio}

Abordemos ahora la cuestión del quantum indemnizatorio, problema que conviene estudiar, recordando una vez más que tal apartado no se refiere a la indemnización del beneficio definitivo frustrado o el mal no evitado, sino precisamente a la oportunidad perdida, que, como bien específico, con propia entidad ontológica, debe ser objeto de un tratamiento indemnizatorio particular. La circunstancia de que en definitiva la magnitud de la pérdida de oportunidad esté en relación con el daño sobrevenido, o con mayor razón para quienes creen que se trata de un caso de causalidad incierta, ha conducido en este problema a aplicar, en general, un método probabilístico, por lo que el monto de la indemnización se fija en una cantidad considerablemente menor que el del beneficio final frustrado ${ }^{29}$. La jurisprudencia de nuestros tribunales, en esta materia, ha tenido una actitud que podemos considerar constante. El profesor Munita comenta la forma en que ordinariamente procede la jurisprudencia francesa. A pesar de que en el país galo está el juez facultado para establecer soberanamente la valoración de la oportunidad, éste se atiene a criterios prudenciales de estimación conforme al principio de cálculo que hemos descrito como "la reparación debe ser calculada mediante el producto de la ventaja suprimida y de la probabilidad en realización de la ventaja, aun cuando se deduce de lo señalado por el mismo autor, que los jueces no están obligados a sujetarse en su estimación a los cálculos rigurosos de las probabilidades, ni a tenerse por matemáticos o algebristas. De acuerdo con lo indicado, los valores probabilísticos del beneficio esperado y la identificación de las probabilidades de percibir el beneficio son fundamentales a la hora de la determinación del quantum resarcitorio" 30 .

Si partimos de la base de que a propósito de la oportunidad el resultado final esperado aparece no solamente como posible sino con altas probabilidades de ocurrencia, de no haber mediado el hecho dañoso, esa oportunidad debe ser cuantificada en relación con tal probabilidad, y no sobre el monto del beneficio que finalmente resulta frustrado: el bien susceptible de indemnización es la oportunidad misma y no el total de la ganancia o la ventaja perdidas ${ }^{31}$.

29 Corte Suprema, 3 de diciembre de 2015, Rol 29.365-2014; Corte Suprema, 22 de septiembre de 2016, Rol 9.481-2016; Corte Suprema, 25 de octubre de 2016, Rol 22.751-2015.

30 Munita, M. R., "La pérdida de una chance. Notas desde una perspectiva comparada". Actualidad Jurídica, vol. XIV , n. ${ }^{\circ} 28$, julio 2013, 438, disponible en [www.actualidadjuridicaudd.cl/revistas/revistaactualidadjuridica-no-28/].

31 Trigo Represas, F. A., op. cit., 263. 


\section{B. La pérdida de oportunidad y los otros daños indemnizables}

Debemos entrar a estudiar la relación que existe entre el daño que significa la pérdida de oportunidad y los demás daños susceptibles de indemnización, como son el daño emergente, el lucro cesante y el daño moral.

Analicemos ante todo el caso relativo al daño emergente. De acuerdo con lo que hemos sostenido hasta ahora, en el sentido de que la oportunidad tiene categoría autónoma, por lo que su pérdida supone un detrimento actual del patrimonio, puede estimarse que su configuración es muy próxima al daño emergente, ya que ambos detrimentos suponen un menoscabo actual en el patrimonio de la víctima del daño. Sin embargo, pese a tales semejanzas, consideramos que las diferencias son notables, significativas y terminantes, y ellas se advierten en tres planos distintos, que son: el bien susceptible de ser dañado, la causalidad en uno y otro supuesto y la indemnización reparatoria del daño. Así, el detrimento patrimonial en el caso del daño emergente recae sobre una parte tangible del patrimonio, y es posible determinar con certeza la naturaleza y cuantía del daño; en el caso de la oportunidad, en cambio, el daño recae sobre un bien que no es tangible, puesto que consiste en la posibilidad de acceder a una ventaja o evitar un menoscabo, por lo que supone el análisis probabilístico respecto de su condición de "seria" y "real". Como consecuencia de ello, la exigencia de causalidad en el daño emergente es terminante y rigurosa, al paso que en el supuesto de la oportunidad, cualquiera que sea la relación entre su pérdida y la conducta del victimario, no puede dejar de relacionarse con lo que hemos llamado el daño definitivo. Finalmente, vemos cómo la determinación del quantum en el daño emergente se establece por la efectiva pérdida económica que sufrió la víctima, mientras que en el caso de la oportunidad se recurre a un cálculo probabilístico cuya consecuencia conduce a rebajar la cuantía que significa o significaría el daño definitivo ${ }^{32}$.

Las diferencias entre la pérdida de oportunidad y el lucro cesante son más claras ya que si éste, como enseña el profesor Corral, consiste en "la frustración de una legítima utilidad que hubiera incrementado el patrimonio de no haber sucedido el hecho dañoso" 33 , el patrimonio de la víctima no sufre disminución actual, pero no se incrementa conforme a la perspectiva cierta de ganancia que existía. En el caso de la oportunidad perdida, el daño consiste propiamente no en un detrimento futuro, el "daño definitivo", sino en el perjuicio actual que se causa por privar a la víctima de esa posibilidad seria y real que tenía de obtener un beneficio o evitar un daño. En este sentido se puede decir que ambos daños se parecen en cuanto significan la pérdida de un beneficio futuro, pero difieren claramente, ya que la pérdida de oportunidad representa un daño actual, en tanto que el lucro cesante es un daño cierto que se producirá en el futuro.

32 Véase al respecto: Chaia, R. A. y Prévôt, J. M., op. cit., 54.

33 Corral TAlciani, H., Lecciones de responsabilidad civil extracontractual, Legal, segunda edición actualizada, Chile, 2013, 142. 
Más complicada es la relación que existe entre la pérdida de oportunidad y el daño moral. Se ha dicho atinadamente que puede entenderse por daño moral todo aquel que no es patrimonial ${ }^{34}$. La pérdida de la oportunidad, conforme a lo que venimos exponiendo, sería un desmedro patrimonial, en cuanto se determina el valor económico de una ocasión perdida como resultado de la negligencia de un tercero, pero no obstante debemos tener en cuenta que la consecuencia dañosa de esa pérdida, el tantas veces mencionado daño definitivo, puede ser la muerte de una persona u otro daño que no resulta propio calificar como patrimonial, y tal es lo que precisamente sucede en la sentencia que comentamos. Con todo, consideramos que aun en estos casos la pérdida de oportunidad no cambia su naturaleza patrimonial, ya que lo que se persigue y la indemnización que se pretende es precisamente a propósito de aquel referido daño concreto, y no las repercusiones extrapatrimoniales que pueda tener. Tales consideraciones resultan básicas si se quiere evitar un mal frecuente en la práctica de nuestros tribunales, vicio que consiste en morigerar la indemnización por daño moral, so pretexto de haberse accedido a indemnizar la pérdida de oportunidad, como si los dos tipos de indemnización fueran de la misma especie ${ }^{35}$.

Creemos haber presentado convenientemente, aunque sea de modo esquemático, las cuestiones doctrinarias fundamentales relativas al caso del maremoto tomado en calidad de referencia. Solo nos falta agregar que las reflexiones de rango teórico enumeradas no parecen modificadas o condicionadas en medida alguna por el hecho de que versen a propósito de una sentencia recaída sobre demanda que han promovido víctimas de rebote.

\section{Consideraciones finales}

Son de público conocimiento los hechos acaecidos a propósito del terremoto y posterior maremoto del año 2010 en la zona sur de Chile.

Las desastrosas consecuencias dieron paso a un importante número de demandas de familiares de las víctimas en contra del Fisco.

Es así como a propósito de los veredictos de los jueces hemos extraído una nueva manera de resarcir los daños llamada "pérdida de la chance", que se suma a las clásicas formas de resarcimiento que conocemos.

En este sentido, a nuestro juicio, los iudicata han sido fallados de manera correcta, de lo contrario, por el evidente problema de causalidad que suscitaban los hechos, las víctimas corrían el riesgo de quedar sin indemnización.

De esta manera, la chance le aplica un correctivo a la indemnización. Esta forma de indemnizar no opera resarciendo completamente el daño sino solo a aquella parte que apunta a la "probabilidad-posibilidad". 
Sin embargo, frente a esta nueva partida indemnizatoria habría que precisar ciertos alcances, debido a que no siempre resultará conveniente impetrarla.

En torno a lo expuesto cabe preguntarse si resulta conveniente indemnizar chances perdidas, en circunstancias en que puede traducirse en la mejor defensa del negligente para reducir el monto de la condena indemnizatoria a la que se expone.

En ese sentido, ¿podría el juez, vía pérdida de una chance, rechazar la indemnización de daños finales (que bien podría indemnizar mediante el recurso a las presunciones), ordenando reparaciones intermedias o de oportunidades (chances)?

Responder a estas interrogantes será una ardua tarea a la que deben contribuir todos los operadores jurídicos. Particularmente tendrán el desafío los jueces, con miras a encauzarla correctamente para que nuestros temores expresados en las interrogantes plasmadas no se concreten.

\section{Referencias}

BÁRCIA, R., Diccionario General Etimológico de la lengua española, voz “oportuno”, “oportunidad", 1881.

Barros Bourie, E., Tratado de responsabilidad extracontractual, Editorial Jurídica de Chile, primera edición, Chile, 2007, 236-237.

Contreras, M. y Winckler, P., "Pérdidas de vidas, viviendas, infraestructura y embarcaciones por el tsunami del 27 de febrero de 2010 en la costa central de Chile". Obras y proyectos, 14, 2013, 6-19.

Corral Talciani, H., Lecciones de responsabilidad civil extracontractual, Legal, segunda edición actualizada, Chile, 2013, 142.

Chaia, R. A. y Prévôt, J. M., Pérdida de chance de curación, Astrea, Argentina, 2007,45 .

Díez Schwerter, J. L., “¿Es imprevisible un sismo en Chile?”, Revista del Abogado, n. ${ }^{\circ} 48,2010,2-7$.

Elorriaga de Bonis, F., "Del daño por repercusión o rebote", Revista Chilena de Derecho, vol. 26, n. ${ }^{\circ}$ 2, 1999, 369-398.

Farfarello Galletti, A. F., Responsabilidad del Estado: terremoto del 27 de febrero de 2010, Santiago de Chile, Rubicón Editores, 2019.

Munita M., R., "La pérdida de una chance. Notas desde una perspectiva comparada", Actualidad Jurídica, vol. XIV , n. ${ }^{\circ}$ 28, julio de 2013. 
Ríos Erazo, I. y Silva Goñi, R., Responsabilidad civil por pérdida de la oportunidad, Chile, Editorial Jurídica de Chile, 2014.

TAPIA Rodríguez, M., "Pérdida de la chance: ¿un perjuicio indemnizable en Chile?”, en Elorriaga de Bonis, F. (coord.), Estudios de Derecho Civil viI, Jornadas Nacionales de Derecho Civil, Viña del Mar, 2011, Legal Publishing Chile.

TAPIA Rodríguez, M., "Pérdida de una chance. Su indemnización en la jurisprudencia chilena", Revista de Derecho n. ${ }^{\circ}$ 2, 2012.

Trigo Represas, F. A., Pérdida de chance, Astrea, Argentina, 2008. 